\title{
METHOD OF EVALUATION A BASIC MECHANIC PROPERTY OF CARTILAGE
}

\author{
Vojtech Havlas ${ }^{2}$, Ivan Dylevský. ${ }^{1}$, Petr Mašát ${ }^{2}$ \\ ${ }^{1}$ Department of Pre-clinical Subjects JU ZSF České Budějovice \\ ${ }^{2}$ Department of Orthopaedic Surgery Charles University, 2 ${ }^{\text {nd }}$ Medical Faculty
}

\begin{abstract}
Many osteoarthritis animal models have been studied, attempting to model these findings after those of humans. an animal model that could develop naturally occurring osteoarthritis in a manner similar to humans would be of great benefit for future research.

Studies with cynomologous macaques have shown that these animals develop naturally occurring osteoarthritis similar to that in humans. The goal of the current project was to examine the basic biomechanical characteristics of normal cynomologous macaque distal femoral articular cartilage as well as to develop a proper biomechanical testing protocol.

Testing was performed with the EnduraTEC machine. Using a $0.5 \mathrm{~mm}$ radius non-permeable cylindrical indenter, instantaneous linear ramp compressions were applied to the cartilage specimens at a rate of 10 $\mathrm{mm} / \mathrm{sec}$ to a depth of $0.15 \mathrm{~mm}$. The thickness of the articular cartilage specimen was determined by using the needle probe. A load deformation curve was obtained and analyzed to determine the thickness. Using a linear elastic model, we assumed that compression was instantaneous. Young's Modulus [N/ $\mathrm{mm} 2=\mathrm{MPa}$ ] was calculated by plotting stress (load/cross-sectional area) vs. strain (displacement/ thickness) and calculating the equation of the line. Stiffness $[\mathrm{N} / \mathrm{mm}]$ was obtained by plotting load vs. displacement. Both were reached calculating the best-fit line form the speed compression ramp.

Only one previous published experiment attempted to characterize the biomechanical properties of normal cynomologous macaque monkey articular cartilage. Our study attempted to use a more simplified approach to calculate Young's modulus and stiffness. By assuming that the compression was instantaneous and using an impermeable indenter we were able to calculate what we feel are important biomechanical properties of articular cartilage.
\end{abstract}

Key words: monkey, cartilage, mechanic property, stiffness

\section{INTRODUCTION}

The purpose of our research was to develop a biomechanical testing protocol for cynomologous macaque (Macaca Fascicularis) articular cartilage. This pilot data will be used to guide future research projects with cynomologous macaque monkey knee articular cartilage.

Osteoarthritis is a debilitating disease, which is the most frequent cause of disability in people older than 65 and the major reason for a huge number of total hip and total knee replacements (Praemer et al. 1992a). In fact, the number of people developing osteoarthritis has increased approximately by 750,000 each year since 1990 in the United States only (Praemer et al. 1992b). With the aging baby boom generation, these numbers will increase in the future all around the world.
Osteoarthritis is frequently characterized as a failure in articular cartilage. Articular cartilage provides the smooth bearing surfaces in freely moving synovial joints and plays an important role in dissipating loads in joints due to rotational and translational motion. Characteristics of osteoarthritis include cartilage degeneration, thickening of the subchondral bone, and the formation of marginal osteophytes (Hernborg a Nilsson 1973). These changes lead to pain, stiffness, and decreased mobility.

The widespread effects of osteoarthritis and the limited ability of articular cartilage to selfrepair (hunter 1995) have increased research interest in developing a method of cartilage regeneration and joint resurfacing. Recent procedures such as autologous chondrocyte implantation (ACI) 
have been developed, and while short-term studies look encouraging (Bentley et al. 2003, Brittberg et al. 1994, Yates 2003), to our knowledge there are no long-term outcome studies in the literature confirming perfect results using this method. Other methods developed include abrasion chondroplasty (Johnson 1996), microfracture (Steadman et al. 1997), and subchondral drilling (Pridie 1959). These all lead to a fibrous cartilage covering which is not similar to hyaline cartilage found in normal, healthy joints. Another drawback includes a limit to the size of lesions that can be repaired.

Many osteoarthritis animal models have been studied, attempting to model these findings after those of humans (Altman a Dean 1990, Bendele 1987, Herzog et al. 1998, Schwartz 1987). However, researchers must be careful when comparing these findings to humans (Warskyi a Hukins 1990). Many of these models use chemical or surgical manipulations of the joints in order to induce the necessary osteoarthritic changes. These changes do not fully simulate the naturally developing process of osteoarthritis seen in most humans. Therefore, an animal model that could develop naturally occurring osteoarthritis in a manner similar to humans would be of great benefit for future research.

Studies with cynomologous macaques have shown that these animals develop naturally occurring osteoarthritis similar to that in humans (Carlson et al. 1994). Detailed histologic, radiographic, and immunohistochemical studies have also shown a high frequency of lesions in the knee joints of these animals (Carlson et al. 1994, Carlson et al. 1995). There have been no studies of cynomologous macaques which characterize the biomechanical properties of their naturally occurring osteoarthritis. This is a future research direction that we plan on pursuing. Pilot data from this study will be used to direct that study.

The goal of the current project was to examine the biomechanical characteristics of normal cynomologous macaque distal femoral articular cartilage.

\section{MATERIALS AND METHODS}

A medial parapatellar incision was performed on 6 cynomologous macaque knees followed subsequently by a capsulotomy taking care to avoid damaging the distal femoral articular cartilage. The ACL, PCL, and remaining structures were transected to allow access to the femoral condyles. An Arthrex (Naples, FL) 5mm coring reamer was placed over the weight bearing areas of the medial and lateral femoral condyles and the cartilage and subchondral bone were taken perpendicular to the articular cartilage to a depth of $5 \mathrm{~mm}$. The harvested specimens were trimmed with a 15 blade so the cartilage surface was parallel with the subchondral bone. The medial and lateral specimens were then fixed to a labeled petri dish using Super Glue. The samples were covered with normal saline and frozen overnight. The day of the testing they were removed and allowed to thaw completely before testing began.

Testing was performed with the EnduraTEC machine. Using a $0.5 \mathrm{~mm}$ radius non-permeable cylindrical indenter, instantaneous linear ramp compressions were applied to the cartilage specimens at a rate of $10 \mathrm{~mm} / \mathrm{sec}$ to a depth of 0.15 $\mathrm{mm}$. Compression was held constant at this depth for $10 \mathrm{sec}$. Two hundred scans per second were recorded. We repeated the above two additional times for a total of three cycles. After performing the linear ramp compressions with the cylindrical indenter, it was replaced with a needle probe. The probe was used to determine the thickness of the articular cartilage specimen. A load of $7.5 \mathrm{~N}$ was applied to the cartilage and the needle probe was allowed to penetrate the surface of the articular cartilage as well as the subchondral bone. A load deformation curve was then obtained and analyzed to determine the thickness. The above results were imported into an Excel (Microsoft Corp.) spreadsheet, which was then used to calculate the thickness, stiffness, and Young's Modulus.

\section{RESULTS}

Early pilot data obtained from the medial and lateral femoral condyles of a 6 monkey knees is summarized below (Tab 1).

Using a linear elastic model, we assumed that compression was instantaneous. Young's Modulus $[\mathrm{N} / \mathrm{mm} 2=\mathrm{MPa}]$ was calculated by plotting stress (load/cross-sectional area) vs. strain (displacement/thickness) and calculating the equation of the line. Stiffness $[\mathrm{N} / \mathrm{mm}]$ was obtained by plotting load vs. displacement. Both were reached calculating the best-fit line form the speed compression ramp.

The thickness of the specimens was obtained by examining the load deformation curve, determining when the articular cartilage was penetrated, and subtracting that point from the point the subchondral bone was penetrated.

At the time of this writing we have not calculated Poisson's ratio. In subsequent experiments we will find this ratio by repeating our testing protocol with an indenter of a different size using 
original calculation method which is still under development. By to developing this method we try avoid using any mechano-optical measurements to reach Poisson's ratio.

\section{DISCUSSION}

Only one previous published experiment attempted to characterize the biomechanical properties of normal cynomologous macaque monkey articular cartilage (Athanasion et al. 1991). This study used a porous-permeable indenter tip of $1.5 \mathrm{~mm}$ and analyzed their results with the linear KLM biphasic model. Using a permeable tip allows unimpeded fluid exudation into the indenter tip. They obtained an aggregate modulus, Poisson's ratio, and permeability values for three pairs of monkey knees. Poisson's ratio was measured mechano-optically. Results are summarized in Tab 2.

Our study attempted to use a more simplified approach to calculate Young's modulus and stiffness. By assuming that the compression was instantaneous and using an impermeable indenter we were able to calculate what we feel are important biomechanical properties of articular cartilage. In future trials we plan on substituting a beveled indenter in place of the cylindrical indenter. We will use a slightly modified equation to account for a beveled indenter. We feel using a beveled indenter will give us more accurate calculations. Poisson's ratio will also be calculated through the use of two beveled indenters of varying sizes.

Results and methods from this study will be used in a future study with monkey cartilage comparing known histology of different stages of osteoarthritis with the biomechanical characteristics of this cartilage. Questions that we hope to answer include: What occurs first, histological or biomechanical changes in the cartilage? Methods developed for the biomechanical testing could be applicable in future studies with stem cell articular cartilage research as well.

\section{REFERENCES}

Altman RD, Dean DD, 1990: Osteoarthritis research: animal models. Sem Arthritis Rheum. 19 (4 Suppl 1) 21-25.

Athanasion KA, Rosenwasser MP, Buckwalter JA, Malinin TI, Mow VC, 1991: Interspecies comparisons of in situ intrinsic mechanical properties of distal femoral cartilage. J Orthop Res. 9, 330-340.

Bendele AM, 1987: Progressive chronic osteoarthritis in femorotibial joints of partial medial meniscectomized guinea pigs. Vet Pathol. 24, 444 -448 .

Bentley G, Biant LC, Carrington RW, Akmal M, Goldberg A, Williams AM, Skinner JA, Pringle J., 2003: A prospective, randomised comparison of autologous chondrocyte implantation versus mosaicplasty for osteochondral defects in the knee. J Bone Joint Surg Br. 85, 223-30.

Brittberg M, Lindahl A, Nilsson A, Ohlsson C, Isaksson O, Peterson L., 1994.: Treatment of deep cartilage defects in the knee with autologous chrondrocyte transplantation. N Engl J Med. 331, 889-95.

Carlson CS, Loeser RF, Jayo MJ, Weaver DS, Adams MR, Jerome CP, 1994: Osteoarthritis in Cynomologous Macaques: a primate model of naturally occurring disease. J Orthop Res. 12, 331339.

Carlson CS, Loeser RF, Johnstone B, Tulli HM, Dobson DB, Caterson B., 1995: Osteoarthritis in cynomologous macaques II: Detection of modulated proteoglycan epitopes in cartilage and synovial fluid. J Orthop Res. 13, 399-409. Hernborg J, Nilsson BE., 1973: The relationship between osteophytes in the knee joint, osteoarthritis, and aging. Acta Orthop Scand. 44, 6974.

Herzog W, Diet S, Suter E, Mayzus P, Leonard TR, Muller C, Wu JZ, Epstein M, 1998: Material and functional properties of articular cartilage and patellofemoral contact mechanics in an experimental model of osteoarthritis. J Biomechan. 31, 1137-1145.

Hunter W., 1995: Of the structure and disease of articulating cartilages. Clin Orthop. 317, 3-6.

Johnson LL, 1996: Arthroscopic abrasion arthroplasty. In: Mcginty JB, Caspari RB, Jackson RW, Poehling GG, eds. Operative Arthroscopy, ed 2. Philadelphia: Lippincott-Raven. 427-446.

Praemer A, Furner S, Rice DP, 1992a: Medical implants and major joint procedures. Musculoskeletal conditions in the United States. Park Ridge (IL): American Academy of Orthopaedic Surgeons, p. 611-26.

Praemer A, Furner S, Rice DP, 1992b: Medical implants and major joint procedures. Musculoskeletal conditions in the United States. Park Ridge (IL): American Academy of Orthopaedic Surgeons; p. 125-42.

Pridie KH, 1959: A method of resurfacing osteoarthritic knee joints. In: Proceedings of the British Orthopaedic Association. J Bone Joint Surg Br. 41, 618-9.

Schwartz ER, 1987. Animal models: a means to study the pathogenesis of osteoarthritis. J Rheumatol. 14, 101-103. 
Steadman JR, Rodkey WG, Singleton SB, Briggs KK, 1997: Microfracture technique for full-thickness chondral defects: Technique and clinical results. Oper Tech Orthop. 7, 300-304.

Warskyi M, Hukins DW, 1990: Animal models for osteoarthritis - ensuring experimental validity. Br J Rheumatol. 29, 219-221.
Yates JW Jr., 2003: The effectiveness of autologous chondrocyte implantation for treatment of full-thickness articular cartilage lesions in workers' compensation patients. Orthopedics. 26, 295-300.

Vojtěch Havlas et al. Dylevsky@seznam.cz.

\section{PŘíLOHA}

Tab 1: Stiffness and Young's Modulus from cartilage specimen

\begin{tabular}{llcc}
\hline & & $\begin{array}{c}\text { Medial } \\
\text { Condyle }\end{array}$ & $\begin{array}{c}\text { Lateral } \\
\text { Condyle }\end{array}$ \\
\hline Stiffness $(\mathrm{N} / \mathrm{mm})$ & Test1 & 10.3640 & 11.4440 \\
& Test2 & 8.1127 & 8.1443 \\
& Test3 & 7.7295 & 7.0212 \\
& Average & 8.7354 & 8.8698 \\
Young's & Test1 & 7.9172 & 5.7971 \\
Modulus $\left(\mathrm{N} / \mathrm{mm}^{2}\right)$ & Test2 & 6.1974 & 4.1635 \\
& Test3 & 5.9046 & 3.5893 \\
& Average & 6.6731 & 4.5166 \\
\hline
\end{tabular}

Tab 2: Results obtained from a total of 6 medial and 6 lateral condyle specimens

\begin{tabular}{lcccc}
\hline & $\begin{array}{c}\text { Poisson's } \\
\text { ratio }\end{array}$ & $\begin{array}{c}\text { Aggregate } \\
\text { modulus }\end{array}$ & Permeability & $\begin{array}{c}\text { Thickness } \\
(\mathrm{mm})\end{array}$ \\
\hline Medial Condyle & 0.236 & 0.815 & 2.442 & 0.72 \\
Lateral Condyle & 0.236 & 0.778 & 4.187 & 0.57 \\
\hline
\end{tabular}

